\title{
Psicooncología
}

ISSN: 1696-7240

https://dx.doi.org/10.5209/psic.71432

\section{La resiliencia de los padres de niños con cáncer y su importancia en el manejo del estrés y la satisfacción vital}

\author{
Anabel Melguizo-Garín ${ }^{1 *}$; $\mathrm{M}^{\mathrm{a}}$ José Martos-Méndez²; Isabel Hombrados-Mendieta³; Iván \\ Ruiz-Rodríguez ${ }^{4}$
}

Recibido: 9 de enero de 2021 / Aceptado: 1 de marzo de 2021

Resumen. Objetivo: El presente estudio se plantea como objetivo principal analizar cómo la resiliencia de los padres de niños diagnosticados con enfermedad oncológica influye en la percepción de estrés y en la satisfacción vital. Metodología: Participaron 112 padres de niños con patología oncológica que recibían tratamiento en Málaga. Los datos fueron recogidos a través de medidas de autoinforme. Resultados: Existe una relación significativa y negativa entre la resiliencia y el estrés percibido, y una relación significativa y positiva entre la resiliencia y la satisfacción vital. En el estudio por dimensiones de la resiliencia y el estrés, la competencia personal de los padres disminuye el estrés $\mathrm{R}^{2}=0,24 ; \mathrm{F}=$ 12,$12 ; \mathrm{p}<0,0001$ y la dimensión de la resiliencia aceptación de uno mismo aumenta la satisfacción vital $\mathrm{R}^{2}=0,42 ; \mathrm{F}=31,24 ; \mathrm{p}<0,0001$. Conclusiones: $\mathrm{El}$ análisis de la resiliencia a través de sus dimensiones ha permitido conocer el papel que tiene sobre el estrés y la satisfacción vital de los padres con niños con cáncer. Estos resultados pueden tener importantes implicaciones prácticas en el diseño de las intervenciones que mejoren su calidad de vida.

Palabras clave: Padres de niños con cáncer, resiliencia, estrés, satisfacción vital.

\section{[en] Resilence, stress level and life satisfaction in parents of children with cancer}

1 Anabel Melguizo-Garín. Departamento de Psicología Social, Trabajo Social, Antropología Social y Estudios de Asia Oriental. Facultad de Psicología. Universidad de Málaga. Campus Universitario de Teatinos s/n. 29071 Malaga (Spain). https://orcid.org/0000-0003-4285-543X.

E-Mail: anamel@uma.es

2 M José Martos-Méndez. Psicología Social, Trabajo Social, Antropología Social y Estudios de Asia Oriental. Facultad de Psicología. Universidad de Málaga. Campus Universitario de Teatinos s/n. 29071 Malaga (Spain). https://orcid.org/0000-0002-7823-5964

E-Mail:mjmartos@uma.es

3 Isabel Hombrados-Mendieta. Psicología Social, Trabajo Social, Antropología Social y Estudios de Asia Oriental. Facultad de Psicología. Universidad de Málaga. Campus Universitario de Teatinos s/n. 29071 Malaga (Spain). https://orcid.org/0000-0001-7288-1589

E-Mail: mihombrados@uma.es

4 Iván Ruiz-Rodríguez. Psicología Social, Trabajo Social, Antropología Social y Estudios de Asia Oriental Facultad de Psicología. Universidad de Málaga. Campus Universitario de Teatinos s/n. 29071 Malaga (Spain). https:// orcid.org/0000-0003-3726-2197

E-Mail: ivan_dlr@uma.es

Los datos que respaldan los hallazgos de este estudio están disponibles previa solicitud al autor de correspondencia.

* Dirección de correspondencia: Anabel Melguizo Garín. Psicología Social, Trabajo Social, Antropología Social y Estudios de Asia Oriental. Facultad de Psicología. Universidad de Málaga. Campus Universitario de Teatinos s/n. 29071 Málaga (Spain).

E-Mail: anamel@uma.es 


\begin{abstract}
Objective: The main objective of this study is to analyze how the resilience of the parents of children diagnosed with oncological disease influences the perception of stress and life satisfaction. Methodology: 112 parents of children with oncological pathology who received treatment in Malaga participated. Data were collected through self-report measures. Results: There is a significant and negative relationship between resilience and perceived stress, and a significant and positive relationship between resilience and life satisfaction. In the study by dimensions of resilience and stress, the personal competence of parents decreases stress $\mathrm{R} 2=0.24 ; \mathrm{F}=12.12 ; \mathrm{p}<0.0001$ and the dimension of resilience acceptance of oneself increases life satisfaction $\mathrm{R} 2=0.42 ; \mathrm{F}=31.24 ; \mathrm{p}<0.0001$. Conclusions: The analysis of resilience through its dimensions has allowed to know the role it has on stress and life satisfaction of parents with children with cancer. These results can have important practical implications in the design of interventions that improve their quality of life.
\end{abstract}

Keywords: Parents of children with cancer, resilience, stress, life satisfaction.

Sumario. 1. Introducción 2. Método 3. Resultados 4. Método estadístico. 5. Resultados. 6. Discusión 7. Conclusiones 8. Agradecimientos. 9. Referencias

Como citar: Melguizo-Garín A, Martos-Méndez MJ, Hombrados-Mendieta I, Ruiz-Rodríguez, I. La resiliencia de los padres de niños con cáncer y su importancia en el manejo del estrés y la satisfacción vital. Psicooncología 2021; 18: 277-291, https://dx.doi.org/10.5209/psic.71432

\title{
1. Introducción
}

El cáncer infantil es una enfermedad que afecta a la vida del niño enfermo y también a la de su entorno más cercano a nivel psicológico y social ${ }^{(1,2)}$. Son muchas las circunstancias nuevas a las que se enfrenta una familia en esta situación. Entre estas circunstancias podemos encontrar la frecuencia alta de tratamientos complejos y hospitalizaciones del niño enfermo, los efectos secundarios de estos tratamientos, la incertidumbre en el proceso y evolución de la enfermedad, así como el miedo a una hipotética recaída ${ }^{(3)}$. En muy probable que ante estas situaciones descritas los padres puedan percibir niveles elevados de estrés que se mantienen en el tiempo, e incluso una vez superada la enfermedad ${ }^{(4)}$. Entre las situaciones derivadas del tratamiento de sus hijos que más estrés suscitan en los padres encontramos las hospitalizaciones, la comunicación de información de la enfermedad y los tiempos que transcurren entre las pruebas y los diagnósticos.

Todas estas situaciones requieren de un gran esfuerzo por parte de los padres que deben gestionar el estrés que el desempeño de tareas complejas relacionadas con el cuidado del hijo enfermo les provoca ${ }^{(5)}$. Esta situación da lugar a que los padres vean como su vida personal y familiar se resiente y sufren una serie de desajustes que en muchas ocasiones da lugar a sentimientos de tristeza y de falta de energía para llevar la situación. Esto se une a la percepción de estrés y crisis que viven debido al cáncer infantil. La adaptación positiva a la situación se relaciona de manera negativa con el estrés que experimentan los padres de niños con cáncer ${ }^{(6)}$. Existen algunos factores psicosociales que podrían amortiguar el impacto negativo que ocasiona el estrés, concretamente, la satisfacción vital y la resiliencia ${ }^{(7)}$.

Frente a las situaciones de estrés se ha destacado el papel de la resiliencia como una variable relacionada con el afrontamiento positivo a la adversidad y las situaciones estresantes. La resiliencia ha sido definida como la capacidad para adaptarse de manera positiva en contextos en los que existe una adversidad significativa ${ }^{(8,9)}$, o la capacidad de sobreponerse a los contratiempos y funcionar con relativa normalidad a pesar de la 
situación adversa ${ }^{(10)}$. También se la definen como la habilidad de funcionar de manera saludable a nivel físico y psicológico ante hechos potencialmente traumáticos. El concepto de resiliencia, en el ámbito de la investigación relacionada con el cáncer, también se le ha llamado crecimiento postraumático ${ }^{(11)}$, porque las cogniciones y emociones positivas que muchas personas desarrollan ante la adversidad pueden deberse también a la propia vivencia de situaciones traumáticas. Con esta dimensión añadida al concepto podemos definir la resiliencia como la capacidad de potenciar el crecimiento y la maduración personal, sin tener que negar la experiencia adversa vivida y otorgándole un sentido ${ }^{(12)}$. Wagnild y Young ${ }^{(13)}$ definieron la resiliencia como una dimensión de la personalidad que mejora la adaptación de las personas. Sobre este concepto de resiliencia los autores desarrollaron un instrumento para medir la capacidad resiliente y encontraron que la resiliencia correlacionaba de manera positiva con la satisfacción vital y de manera negativa con la depresión ${ }^{(13)}$.

La satisfacción vital parece tener relación con la capacidad resiliente, se ha encontrado una relación positiva entre ambos constructos, a mayor nivel de resiliencia mayor satisfacción vital ${ }^{(14)}$. La satisfacción vital es un aspecto cognitivo que se basa en la valoración global que las personas hacen de sus propias vidas ${ }^{(15)}$. Además, es un constructo multidimensional que está conformado por diferentes dimensiones, familia, trabajo, salud, etc. La vivencia del cáncer tiene importantes repercusiones sobre la satisfacción vital de los padres ${ }^{(16)}$. En este sentido, la calidad de vida de los padres de niños con cáncer se ve afectada de manera negativa por la enfermedad oncológica ${ }^{(17)}$. Las situaciones vividas en este proceso tienen una incidencia alta en la percepción de estrés y también se traducen en una satisfacción vital baja ${ }^{(18)}$.

Son escasos los estudios que se centran en el análisis de las relaciones entre resiliencia y satisfacción vital. Se ha estudiado, entre otras cuestiones relacionadas con las familias con niños con cáncer, la capacidad resiliente de cuidadoras de niños con cáncer y la relación que guardan los tipos de funcionamiento familiares y la resiliencia en padres de niños con cáncer ${ }^{(19,20)}$. Es importante conocer qué impacto tiene la resiliencia en variables como el estrés y la satisfacción vital de los padres de estos niños.

El presente estudio se plantea como objetivo principal analizar cómo la resiliencia de los padres de niños diagnosticados con enfermedad oncológica influye en la percepción de estrés y en la satisfacción vital. Aunque el papel de la resiliencia es una variable ampliamente estudiada en pacientes adultos y menores con cáncer, son escasas las investigaciones que se centran en estudiar la relación de estas variables en los padres de niños con cáncer ${ }^{(21,22)}$.

De acuerdo con los objetivos del estudio las hipótesis que se plantean son:

1. Existe una relación negativa y significativa entre la resiliencia y el estrés.

2. Existe una relación positiva y significativa entre la resiliencia y la satisfacción vital.

3. Mayores niveles de resiliencia predicen menor nivel de estrés y mayor satisfacción vital.

\section{Método}

\section{Participantes}

Participaron en este estudio 112 padres de niños con patología oncológica. La muestra estaba formada por $33,9 \%$ hombres y $66,1 \%$ mujeres, con una media de edad de 41 
años $(\mathrm{DT}=6,93)$. Los padres participantes en el estudio, en su mayoría, estaban casados o vivían en pareja $(88,5 \%)$, estando el resto solteros, divorciados o viudos $(11,5 \%)$. En cuanto al sexo de los niños que padecían la enfermedad, 58\% eran niños y $42 \%$ niñas, y la media de edad fue de $8(\mathrm{DT}=5,02)$. Con respecto al tipo de cáncer, el 53,6\% padecían leucemia, el 9\% sarcoma de Ewing, el 8\% linfoma, el 4,5\% meduloblastoma, el 4,5\% neuroblastoma y, el resto, otros tipos de patologías oncológicas. Por último, el tiempo transcurrido desde el diagnóstico de la enfermedad ha sido el siguiente: el $18,9 \%$ de los niños han sido diagnosticados hace menos de un año, el 23,4\% hace un año, el $19,8 \%$ hace dos años, el $9,9 \%$ hace tres años, el $12,6 \%$ hace 4 años y el resto $15,3 \%$ hace cinco o más años desde el diagnóstico de la enfermedad.

La muestra fue recogida entre los padres y madres de niños y adolescentes que recibían tratamiento en el Hospital Materno-Infantil de Málaga o en el caso de padres de pacientes entre los 15 y 21 años en el Hospital Regional de Málaga (España). La selección de los participantes se realizó contando con todos los padres que quisieron participar de manera voluntaria en el estudio. Podían participar uno o ambos progenitores del niño o adolescente, no se delimitó está cuestión a la hora de seleccionar la muestra. El criterio de inclusión en la muestra del estudio fue: madres, padres o tutores de pacientes entre 0 y 21 años con patología oncológica. Los criterios de exclusión fueron: otros familiares de los pacientes que no fueran progenitores o tutores legales y que el hijo o hija hubiera fallecido.

Tabla 1.Variables sociodemográficas $(n=112)$

\begin{tabular}{lc}
\hline Edad del padre/madre/tutor & $41(6,93)^{\mathrm{a}}$ \\
Número de hijos & $1,98(0,67)^{\mathrm{a}}$ \\
Número de personas a cargo & $2,18(1,15)^{\mathrm{a}}$ \\
Edad del hijo/a enfermo/a & $8(5,02)^{\mathrm{a}}$ \\
Género del padre/madre/tutor & $\%(\mathrm{~N})$ \\
Hombre & $33,9(38)$ \\
Mujer & $66,1(74)$ \\
\hline Estado civil & $3,6(4)$ \\
\hline Soltero/a & $80,4(90)$ \\
Casado/a & $3,6(4)$ \\
Divorciado/a & $3,6(4)$ \\
Separado/a & $0,9(1)$ \\
Viudo/a & $1,8(2)$ \\
Pareja de hecho & $6,3(7)$ \\
Vive en pareja & $31,3(35)$ \\
\hline Nivel académico & $33(37)$ \\
\hline Universitarios & $11,6(13)$ \\
Ciclo profesional & $22,3(25)$ \\
Bachillerato & $1,8(2)$ \\
Enseñanza obligatoria & \\
Otros & \\
\hline
\end{tabular}




\begin{tabular}{lc}
\hline Situación laboral & \\
\hline Funcionario & $13,4(15)$ \\
En activo, por cuenta propia & $11,6(13)$ \\
En activo, contratado & $31,3(35)$ \\
Desempleado & $33(37)$ \\
Tareas domésticas & $10,7(12)$ \\
\hline Género del hijo/a enfermo/a & \\
\hline Niño & $58(65)$ \\
Niña & $42(47)$ \\
\hline Tipo de cáncer que padece el hijo/a enfermo/a \\
\hline Leucemia & $53,6(60)$ \\
Sarcoma de Ewing & $8,9(10)$ \\
Linfoma & $8(9)$ \\
Meduloblastoma & $4,5(5)$ \\
Neuroblastoma & $4,5(5)$ \\
Rabdomiosarcoma & $2,7(3)$ \\
Hepatoblastoma & $2,7(3)$ \\
Astrocitoma & $1,8(2)$ \\
Otros & $13,4(15)$ \\
\hline Tiempo transcurrido desde el diagnóstico & \\
\hline Menos de 1 año & $18,9(22)$ \\
1 año & $23,4(26)$ \\
2 años & $19,8(22)$ \\
3 años & $9,9(11)$ \\
4 años & $12,6(14)$ \\
5 años o más & $15,3(17)$ \\
\hline a Media (Desviación Típica) &
\end{tabular}

\section{Procedimiento}

Los padres participantes en el estudio acudían de manera regular al Hospital Infantil de Málaga y se contactó con ellos directamente en el Hospital (consultas de oncología, hospitalización de día y zona de hospitalización) o en las salas de juegos para los menores enfermos que una ONG local tiene dentro del Hospital para prestar apoyo a familias con niños con cáncer. El personal sociosanitario informaba a los progenitores de la existencia del estudio y aquellos que decidían participar de manera voluntaria acudían a punto de recogida de datos que se habilitó dentro del Hospital, otro medio para informar de la posibilidad de participar fue a través de un cartel informativo situado en la planta de oncología. Los participantes recibieron un consentimiento informado sobre el procedimiento que se llevaría a cabo. El estudio cuenta con la aprobación del Comité Ético de Investigaciones Científicas de la Junta de Andalucía (España), CEI 2017. Los padres participantes, tras la firma del consentimiento informado, pudieron elegir dos formas de completar el instrumento: en papel en alguna de sus visitas al Hospital, o en formato on-line a través de un 
modelo de instrumento informatizado que una vez recogido se añadía de manera anónima y automática a una base de datos.

\section{Instrumentos}

- Cuestionario sociodemográfico. Cuestionario sociodemográfico en el que se pregunta por datos acerca del sexo, edad, estado civil, nivel de estudios, situación laboral, número de hijos y número de personas a cargo con respecto a los padres participantes. También se pegunta por el hijo enfermo: sexo, edad, tipo de cáncer y tiempo del diagnóstico.

- Cuestionario de resiliencia. Escala de resiliencia de Wagnild y Young (1993) ${ }^{(13)}$, para este estudio hemos utilizado la adaptación española de Heilemann et al. (2007) (23). Esta escala está compuesta por 25 preguntas tipo Likert, los valores oscilan entre 1 "totalmente en desacuerdo" y 7 "totalmente de acuerdo". La escala la conforman dos dimensiones, la primera denominada competencia personal consta de 17 ítems en los que se recogen preguntas que hacen referencia a la fortaleza personal, la autosuficiencia, la independencia, la invencibilidad, el dominio, el ingenio y la perseverancia (p.e. "Dependo de mí mismo/a más que de cualquier otra persona"). La segunda dimensión llamada aceptación de uno mismo y de la vida está compuesta por 8 ítems y se relacionan con la capacidad de adaptación, la flexibilidad y una visión equilibrada de la vida, (p.e. "Acepto que haya personas a las que yo no les caiga bien”)(13). Los ítems están redactados de manera positiva y mayores puntuaciones en la escala indican mayor capacidad resiliente. El alfa de Cronbach es de 0,95 .

- Inventario Pediátrico para Padres (PIP). Inventario Pediátrico para Padres (PIP), versión española del Pediatric Inventory for Parents de Streisand et al. $(2001)^{(24)}$ adaptación y validación de Del Rincón, Remor y Arranz (2007) ${ }^{(25)}$. Este cuestionario evalúa el estrés que generan las situaciones a las que se enfrentan diariamente los padres de niños con enfermedad oncológica. Se compone de una escala de frecuencia y otra escala de esfuerzo. Un ejemplo de ítem de la escala de frecuencia sería: “¿Con qué frecuencia tiene dificultades para dormir?”, y de la escala esfuerzo: “¿Cuánto esfuerzo le implica estar con su hijo/a durante las pruebas médicas y los tratamientos?". Cada una de las escalas consta de 42 preguntas relacionadas con situaciones a las que los padres han de enfrentarse durante la enfermedad del hijo, éstos tienen que contestar, en primer lugar, con qué frecuencia ocurre dicho ítem pudiendo elegir entre cinco opciones de respuesta en la que " 1 " es nunca y " 5 " es muy a menudo; y, posteriormente, cuánto esfuerzo le supone dicha afirmación, eligiendo entre 5 opciones en la que " 1 " es nada y " 5 " es muchísimo. El alfa de Cronbach es de 0,95.

- Escala de satisfacción con la vida. Escala de satisfacción con la vida de Pavot y Diener $(1993)^{(26)}$. Esta escala ofrece un índice general de satisfacción con la vida, entendida ésta como un constructo general de bienestar subjetivo. Es una escala unidimensional compuesta por 5 ítems que se corresponden con una escala tipo Likert de 7 puntos $(1=$ completamente en desacuerdo y $7=$ completamente de acuerdo). Un ejemplo de ítem de esta escala sería: "En la mayoría de las cosas, mi vida está cerca de mi idea”. El alfa de Cronbach es de 0,87. 


\section{Método estadístico}

Los análisis estadísticos se llevaron a cabo con el SPSS (v.24). Primero se realizó un análisis descriptivo para conocer las características de la muestra, así como los niveles de resiliencia, estrés y satisfacción vital de los padres (tabla 2), no se detectaron valores perdidos. Para conocer el grado de relación existente entre las diferentes variables (resiliencia, estrés y satisfacción vital), se llevó a cabo un análisis correlacional de Pearson (tabla 3). Para conocer si existían diferencias significativas entre los padres y las madres participantes en el estudio en los niveles de resiliencia, estrés y satisfacción vital atendiendo al género se realizó una prueba $\mathrm{T}$ para muestras independientes (tabla 4). Y por último se realizó un análisis de regresión lineal múltiple para indagar más en las relaciones existentes entre las variables del estudio, incluyendo además algunas variables sociodemográficas y clínicas que pueden ser relevantes junto a la resiliencia en la percepción de estrés y la satisfacción vital de los padres. (tabla 5 y 6). Las variables que se tuvieron en consideración en la ecuación de regresión fueron aquellas que alcanzaron significación estadística $(\mathrm{p}<0,05)$.

\section{Resultados}

\section{Análisis descriptivos y correlaciones}

Con el objetivo de conocer el nivel de resiliencia de los padres, así como de estrés y satisfacción vital, se han realizado los análisis descriptivos que se ven en la tabla 2. Los resultados indican que los padres tienen un nivel de resiliencia medio-alto. Además, manifiestan sentir un nivel de estrés medio relacionado con el afrontamiento a diferentes situaciones relacionadas con la enfermedad de sus hijos. Con respecto a la satisfacción vital, los padres informan de un nivel medio-bajo.

Tabla 2. Media y desviación típica de resiliencia, estrés y satisfacción vital.

\begin{tabular}{lccc}
\hline & $\mathrm{N}$ & $\mathrm{M}$ & $\mathrm{D} . \mathrm{T}$. \\
\hline Resiliencia & 112 & 4,94 &, 85 \\
PIP estrés & 112 & 3,01 &, 67 \\
Satisfacción vital & 112 & 3,53 & 1,17 \\
\hline
\end{tabular}

En el siguiente apartado se describen las relaciones existentes entre la resiliencia de los padres con el estrés y la satisfacción vital. Se han realizado análisis de correlaciones de Pearson, los resultados se recogen en la tabla 3. Los resultados muestran que existe una relación negativa y significativa entre la resiliencia y el estrés que los padres experimentan al afrontar diferentes situaciones relacionadas con la enfermedad de sus hijos. Existe una relación positiva y significativa entre la resiliencia y la satisfacción vital de los padres. Además, se ha hallado una relación significativa y negativa entre el nivel de estrés de los padres y la satisfacción vital. 
Tabla 3. Correlaciones de la resiliencia con el estrés y la satisfacción vital

\begin{tabular}{lccc}
\hline & Resiliencia & Estrés & $\begin{array}{c}\text { Satisfacción } \\
\text { vital }\end{array}$ \\
\hline Resiliencia & 1 & & \\
Estrés &,$- 384(*)$ & 1 & 1 \\
Satisfacción vital &, $638(* * *)$ &,$- 279(* *)$ & 1 \\
\hline
\end{tabular}

${ }^{*} p<, 05 ; * *_{p}<, 01 ; * * * p<, 001$

\section{Diferencias de medias}

Para conocer si existen diferencias significativas entre los padres participantes en el estudio atendiendo al género de éstos, se realizó una prueba $T$ para muestras independientes en la que las variables de prueba fueron la resiliencia, el estrés y la satisfacción vital. La variable de agrupación fue el género de los participantes en el estudio. Los resultados indican que no existen diferencias significativas con respecto al género de los participantes en cuanto a la resiliencia, el estrés y la satisfacción vital (tabla 4).

Tabla 4. Diferencias de medias de la resiliencia, del estrés y de la satisfacción vital según el género

\begin{tabular}{ccccccc}
\hline & \multicolumn{2}{c}{ Resiliencia } & \multicolumn{2}{c}{ Estrés } & \multicolumn{2}{c}{ Satisfacción vital } \\
\cline { 2 - 6 } & $\mathrm{M}(\mathrm{DT})$ & $\mathrm{p}$ & $\mathrm{M}(\mathrm{DT})$ & $\mathrm{p}$ & $\mathrm{M}(\mathrm{DT})$ & $\mathrm{p}$ \\
\hline Género & & & & & & \\
Hombre & $5,05(0,49)$ &, 402 & $2,85(0,68)$ &, 076 & $3,80(0,99)$ &, 081 \\
Mujer & $4,85(1,05)$ & & $3,09(0,66)$ & & $3,39(1,24)$ & \\
\hline
\end{tabular}

\section{Análisis de regresión múltiple}

Para conocer mejor la relación existente entre la resiliencia de los padres con el estrés y la satisfacción vital, se ha realizado un análisis de regresión lineal múltiple. Se pretende analizar si la resiliencia de los padres actúa como predictora del estrés que sufren, así como de la satisfacción vital de éstos. La resiliencia ha sido considerada en sus dos dimensiones competencia personal y aceptación de uno mismo. Además en el análisis de regresión se han incluido variables de tipo sociodemográficas y clínicas para conocer la relación que pueden tener con el estrés percibido y la satisfacción vital de los padres. En concreto se han considerado para este análisis como posibles variables predictoras el género de los padres, la edad de los padres, el número de hijos, el número de personas a cargo, el género del hijo enfermo, la edad del hijo enfermo y el tiempo transcurrido desde el diagnóstico. Se ha realizado un 
análisis de regresión en el que las variables dependientes han sido el estrés percibido por los padres y la satisfacción vital. Han actuado como variables independientes la resiliencia (competencia personal y aceptación de uno mismo) y las variables sociodemográficas comentadas. El método utilizado para este análisis de regresión múltiple es el de pasos hacia atrás "Backward Stepwise Regression".

Con respecto a la variable estrés, es la dimensión competencia personal de la resiliencia y el tiempo transcurrido desde el diagnóstico de la enfermedad del hijo, las dos variables que mejor la predicen (tabla 5). La ecuación de regresión tiene una $\mathrm{R}^{2}=0,29 ; \mathrm{F}=7,09$; para la competencia personal $\mathrm{p}=0,002$, y para el tiempo transcurrido desde el diagnóstico $p=0,022$. Mayores niveles de competencia personal y mayor tiempo transcurrido desde el diagnóstico predicen menores niveles de estrés percibido por parte de los padres.

En cuanto a la satisfacción vital, es la dimensión aceptación de uno mismo de la escala de resiliencia la que ha resultado ser significativa. En este caso la dimensión aceptación de uno mismo aumenta de manera significativa la satisfacción vital $\left(\mathrm{R}^{2}=\right.$ 0,$42 ; \mathrm{F}=31,24 ; \mathrm{p}<0,0001$ ) (tabla 5).

Tabla 5. Coeficientes estandarizados de regresión de las dimensiones competencia personal y aceptación de uno mismo de la escala de resiliencia y las variables sociodemográficas sobre el estrés y la satisfacción vital

\begin{tabular}{|c|c|c|c|c|c|}
\hline & B & $\mathrm{t}$ & $\mathrm{p}$ & $\mathrm{R}^{2}$ & $\mathrm{~F}$ \\
\hline Competencia personal &,- 45 & $-3,27$ &, $002 * *$ & & \\
\hline Tiempo desde el diagnóstico &,- 33 & $-2,39$ &, $022 *$ & & \\
\hline Variable dependiente: Estrés & & & & ,29 & 7,09 \\
\hline Aceptación de uno mismo & ,65 & 5,59 &, $000 * *$ & & \\
\hline Variable dependiente: Satisfacción vital & & & &, 42 & 31,24 \\
\hline
\end{tabular}

Nota 1. **p $\leq 0.01 ; * p \leq 0.05$

Variables dependientes: Estrés (escala PIP)

Satisfacción vital

También se ha realizado un análisis de regresión lineal múltiple a través del método intro teniendo en cuenta las dimensiones de la variable estrés. El objetivo es conocer con mayor profundidad qué dimensión de la resiliencia predice mejor cada una de las dimensiones del estrés. La variable estrés se ha dividido en las dos dimensiones, frecuencia y esfuerzo y aparecen como variables dependientes. La resiliencia que actúa como variable independiente se ha mantenido en este análisis en sus dos dimensiones, competencia personal y aceptación de uno mismo.

El análisis realizado indica que la dimensión competencia personal de la resiliencia reduce de manera significativa el esfuerzo que los padres tienen que realizar con respecto a la enfermedad de sus hijos $\left(\mathrm{R}^{2}=0,24 ; \mathrm{F}=12,12 ; \mathrm{p}<0,0001\right)$, la relación entre la dimensión frecuencia del estrés y competencia personal no ha resultado significativa. La relación entre la dimensión esfuerzo del estrés y frecuencia del estrés no ha resultado ser significativa con respecto a la dimensión aceptación de uno mismo de la resiliencia (tabla 6). 
Tabla 6. Coeficientes estandarizados de regresión de las dimensiones competencia personal y aceptación de uno mismo de la escala de resiliencia sobre las dimensiones del estrés (frecuencia y esfuerzo)

\begin{tabular}{lrrrrr}
\hline & $\mathrm{B}$ & $\mathrm{t}$ & $\mathrm{p}$ & $\mathrm{R}^{2}$ & $\mathrm{~F}$ \\
\hline $\begin{array}{l}\text { Competencia personal } \\
\text { Variable dependiente: Estrés frecuencia }\end{array}$ &,- 27 & $-1,73$ &, 091 & & \\
$\begin{array}{l}\text { Competencia personal } \\
\text { Variable dependiente: Estrés esfuerzo }\end{array}$ &,- 02 & $-3,48$ &, $001^{* *}$ &, 05 & 2,99 \\
$\begin{array}{l}\text { Aceptación de uno mismo } \\
\text { Variable dependiente: Estrés frecuencia }\end{array}$ &,- 25 & $-1,63$ &, 111 &, 24 & 12,12 \\
$\begin{array}{l}\text { Aceptación de uno mismo } \\
\text { Variable dependiente: Estrés esfuerzo }\end{array}$ & $, 02,69$ &, 496 &, 04 & 2,65 \\
\hline
\end{tabular}

Nota $1 . * * p \leq 0.01 ; * p \leq 0.05$

Variables dependientes: Estrés frecuencia (escala PIP)

Estrés esfuerzo (escala PIP)

\section{Discusión}

Los análisis que se han llevado a cabo nos informan de la importancia de conocer cómo se relaciona la resiliencia de los padres de niños enfermos de cáncer con variables tan destacadas en el proceso que están viviendo como son el estrés percibido y la satisfacción vital.

Con respecto a la primera hipótesis planteada, la resiliencia se relaciona con una menor percepción del estrés. Los padres sienten un nivel medio de estrés relacionado con las situaciones derivadas de la enfermedad del hijo (ingresos hospitalarios, realización de tratamientos, comunicación con los profesionales sanitarios, tareas relacionadas con el cuidado y las necesidades de la familia y del hijo, etc.) ${ }^{(27)}$. Esta información es relevante ya que potenciar la capacidad resiliente de los padres puede ser útil para que lleven a cabo una mejor gestión del estrés y de las tareas y rutinas que tienen que desempeñar en su situación. En la línea de estos resultados encontramos estudios como los de Fuenmmeler et al. ${ }^{(6)}$, Harms et al. ${ }^{(7)}$, Huang et al. ${ }^{(8)}$ y Lee et al. ${ }^{(9)}$ entre otros.

Con respecto a la segunda hipótesis planteada, vemos que mayores índices de resiliencia se relacionan con mayor satisfacción vital en los padres. En este sentido asociado a otros contextos también se han descubierto relaciones positivas entre resiliencia y satisfacción vital como en el estudio de Liu et al. ${ }^{(28)}$. En relación al contexto psico-oncológico Zayas et al. ${ }^{(14)}$ también encuentran una relación positiva entre la resiliencia y la satisfacción vital. Los niveles de satisfacción vital de los padres participantes en este estudio son medio-bajos, sin duda este dato puede ir asociado a la compleja situación por la que están pasando. La satisfacción vital es una variable que se relaciona de manera muy estrecha con la autorrealización personal y en situaciones como la del cáncer infantil que conlleva una gran implicación física y psicológica por parte de los progenitores, puede incidir en que éstos vean mermadas sus posibilidades de autorrealización. 
Es importante destacar que los padres informan de unos niveles de resiliencia medioaltos, resultados que van en la línea con estudios previos como el de Navea y Tamayo ${ }^{(20)}$, en el que se revisa el concepto de resiliencia familiar y concluyen que niveles altos de resiliencia se asocian a baja sobrecarga de los cuidadores. Además estos niveles elevados de resiliencia se suelen asociar al concepto de crecimiento postraumático ${ }^{(29)}$, ya que en momentos de crisis personal y en situaciones potencialmente traumáticas como puede ser en este caso la vivencia del padecimiento de cáncer por parte de un hijo, genere en los padres un proceso de adaptación positiva y búsqueda de sentido a la situación vivida. En este sentido investigaciones previas como la de Guil et al. ${ }^{(30)}$, concluyen que trabajar la capacidad resiliente, en este caso en mujeres que padecen cáncer, puede contribuir a mejorar el afrontamiento y bienestar de éstas. Sin duda, este es un aspecto muy positivo en el proceso de afrontamiento que están viviendo los padres, en este caso por el enfermedad oncológica de un hijo. Esta cuestión tiene unas implicaciones prácticas importantes, autores como Ochoa et al. ${ }^{(31)}$ destacan en esta línea que el fomento de la capacidad resiliente a través de la psicología positiva aplicada al cáncer puede reducir el malestar psicológico.

En la tercera hipótesis vemos precisamente la relación que esta capacidad resiliente de los padres tiene con el estrés y la satisfacción vital. La dimensión de la resiliencia "competencia personal" predice menores niveles de estrés y la dimensión "aceptación de uno mismo" mayores niveles de satisfacción vital en los padres, por lo que la resiliencia puede jugar un papel muy beneficioso en la adaptación al cáncer infantil por parte de los padres. Autores como Matos y Mercerón ${ }^{(19)}$ concluyen en estudios previos que uno de los aspectos más relevantes en los padres con hijos con cáncer es el malestar y angustia que pueden sentir y que el fomento de la capacidad resiliente puede beneficiar a estos padres. Por otro lado Martín y Tamayo ${ }^{(32)}$ en la línea de estos resultados encuentran que la resiliencia puede ser un recurso psicológico amortiguador de problemas derivados de la situación que están viviendo.

Es también muy importante destacar que para conocer mejor las relaciones entre estas variables, se ha realizado el análisis por dimensiones, ya que la variable resiliencia se ha estudiado a través de la Escala de Resiliencia de Wagnild y Young $(1993)^{(13)}$ que cuenta con dos dimensiones, competencia personal y aceptación de uno mismo; y la variable estrés también cuenta con dos dimensiones, frecuencia y esfuerzo en el cuestionario utilizado en este estudio, Inventario Pediátrico para Padres (PIP), versión española del Pediatric Inventory for Parents de Streisand et al. $(2001)^{(24)}$. Es interesante resaltar que la dimensión de competencia personal reduce la percepción de esfuerzo que los padres sienten que hacen derivado de la situación del cáncer infantil. Estos resultados pueden ser trasladados a un escenario práctico a la hora de diseñar programas de apoyo a los padres. Potenciar las capacidades resilientes puede mejorar el afrontamiento que las familias hagan de la situación como apuntan estudios previos ${ }^{(19)}$. Y en este estudio en concreto, conocer qué dimensión resulta más efectiva para reducir los niveles de estrés y mejorar la satisfacción vital, es un aspecto interesante.

\section{Conclusión}

Conocer la relación existente entre la resiliencia con el estrés y la satisfacción vital de los padres puede tener importantes implicaciones prácticas en el diseño de las intervenciones que mejoren su calidad de vida. Es necesario el tratamiento de 
los aspectos psicosociales para mejorar la calidad de vida de los padres de niños enfermos con cáncer ${ }^{(33)}$. Para poder planificar actuaciones realmente eficaces en este sentido, se hace necesario conocer qué relación guardan las variables psicosociales implicadas en el proceso $^{(34)}$.

En este estudio podemos encontrar algunas limitaciones que son importantes tener en cuenta. Una de ellas es su diseño transversal. En futuros estudios sería interesante plantear un estudio longitudinal con el que pudiéramos conocer el comportamiento y la relación de estas variables con mayor profundidad y con la perspectiva temporal. Otra cuestión importante es la procedencia de los participantes en el estudio. Aunque no todos son originarios de Málaga (España), sí que todos los padres participantes son de zonas cercanas, este hecho puede limitar la generalización de los resultados a otros contextos y culturas. En relación a las características de la muestra del estudio, han participado en el mismo más madres que padres. Este puede ser uno de los motivos por el cual no hemos encontrado en esta investigación diferencias significativas en los niveles de resiliencia, estrés y satisfacción vital en cuanto al género de los progenitores participantes en el estudio. Autores como Monteagudo et al. ${ }^{(35)}$ en una muestra de pacientes con cáncer encuentran diferencias significativas en resiliencia con respecto al género. En otras investigaciones realizadas en otros contextos sobre el estudio de la resiliencia y el género si se encontraron diferencias significativas en cuanto al género en la resiliencia ${ }^{(36-38)}$ o en alguna dimensión de la resiliencia ${ }^{(39)}$. De cara a futuras investigaciones sería necesario igualar el número de madres y padres participantes ya que de ese modo podremos conocer si existen relaciones entre las variables de manera diferencial. Este hecho es debido a un fenómeno social que ocurre con relativa frecuencia en este ámbito, y es que las madres ejercen un rol más determinante en el cuidado de sus hijos enfermos y suelen participar de manera más activa en las acciones derivadas de dicha situación, incluida la participación en estudios $^{(40)}$. Para futuras investigaciones podría ser también interesante realizar un análisis de las variables del estudio pero atendiendo a factores como las diferentes fases de la enfermedad y los diferentes tipos de cáncer.

También es importante destacar las fortalezas de este estudio. En el mismo se hacen aportaciones novedosas en cuanto a las relaciones que guardan variables psicosociales muy relevantes en el proceso de afrontamiento del cáncer infantil por parte de los padres. Profundizar en las implicaciones que tiene la capacidad resiliente en el estrés percibido y la satisfacción vital tiene un gran valor para mejorar la adaptación y afrontamiento de los padres al proceso de la enfermedad. Además, el análisis por dimensiones de las variables resiliencia y estrés, nos permiten obtener una información más detallada de qué aspectos son los que más inciden en la calidad de vida de los padres. Este estudio puede ser muy útil para optimizar las intervenciones clínicas que se realizan con estos padres y mejorar los protocolos psicosociales de actuación. Son escasos los estudios de tipo cuantitativo que se centran en profundizar en las relaciones entre las variables psicosociales de los padres de niños que padecen cáncer. Los resultados obtenidos son importantes ya que pueden mejorar la tarea diaria de los agentes implicados en la prestación de servicios psicosociales para estas familias. Conocer qué papel juega la capacidad resiliente en el afrontamiento del cáncer infantil por parte de los padres y cómo se relaciona con el estrés que éstos viven y el impacto que puede tener sobre su satisfacción vital, puede ayudar a poner en marcha estrategias terapéuticas que potencien la capacidad resiliente de los padres ya que puede contribuir a una mejor calidad de vida. 


\section{Agradecimientos}

Los autores quieren agradecer su colaboración en este estudio a la Fundación Olivares (Málaga, España) y a los padres que participaron en el mismo.

\section{Referencias bibliográficas}

1. Carlsen LT, Christensen SR, Olesen SP. Adaption strategies used by siblings to childhood cancer patients. Psychooncology 2019; 28: 1438-44. https://dx.doi.org/10.1002/pon.5093

2. Wilford JG, Hopfer S, Wenzel L. Perceptions of changes in clinical, informational, and supportive relationships after end of treatment among parents of young childhood cancer survivors. Psychooncology 2019; 28: 913-19. https://dx.doi.org/ 10.1002/pon.5042

3. Sloper P. Predictors of distress in parents of children with cancer: a prospective study. J Pediatr Psychol 2000; 225: 79-91. https://dx.doi.org/10.1093/jpepsy/25.2.79

4. Bilodeau M, Ma C, Al-Sayegh H, Wolfe J, Bona K. Household material hardship in families of children post-chemotherapy. Pediatr Blood Cancer 2018; 65: e26743. https:// dx.doi.org/10.1002/pbc. 26743

5. Fernández B, Pascual C, Yélamos C. Cáncer infantil. Guía de apoyo para padres. Madrid: AECC, 2006.

6. Fuemmeler BF, Brown RT, Williams L, Barredo J. Adjustment of children with cancer and their caregivers: moderating influences of family functioning. Fam Syst Health 2003; 21: 263-76. https://dx.doi.org/10.1037/1091-7527.21.3.263

7. Harms AA, Cohen L, Pooley JA, Chambers SK, Galvão DA, Newton RU. Quality of life and psychological distress in cancer survivors: The role of psycho-social resources for resilience. Psychooncology 2018; 28: 271-77. https://dx.doi.org/10.1002/pon.4934

8. Huang Y, Huang Y, Bao M, Zheng S, Du T, Wu K. Psychological resilience of women after breast cancer surgery: A cross-sectional study of associated influencing factors. Psychol Health Med 2019; 5: 1-13. https://dx.doi.org/10.1080/13548506.2019.1574353

9. Lee SY, Lee H, Fawcett J, Park JH. Resilience in Koreans with cancer: Scoping review. J Hosp Palliat Nurs 2019. https://dx.doi.org/ 10.1097/NJH.0000000000000543

10. Stephens TM. Nursing student resilience: a concept clarification. Nurs Forum 2013; 48: 125-133. https://dx.doi.org/10.1111/nuf.12015

11. Greup SR, Kaal SEJ, Jansen R, Manten-Horst E, Thong MSY, van der Graaf WTA, et al. Post-traumatic growth and resilience in adolescent and Young adult cancer patients: An overview. J Adolesc Young Adult Oncol 2018; 7: 1-14. https://dx.doi.org/10.1089/ jayao.2017.0040

12. Mundey KR, Nicholas D, Kruczek T, Tschopp M, Bolin, J. Posttraumatic growth following cancer: The influence of emotional intelligence, management of intrusive rumination, and goal disengagement as mediated by deliberate rumination. J Psychosoc Oncol 2018; 37 : 1-22. https://dx.doi.org/10.1080/07347332.2018.1514449

13. Wagnild GM, Young HM. Development and psychometric evaluation of the resilience scale. J Nurs Meas 1993; 1: 165-78.

14. Zayas A, Gómez-Molinero R, Guil R, Gil-Olarte P, Jiménez E. Relación entre la resiliencia y la satisfacción con la vida en una muestra de mujeres con cáncer de mama. IJDP 2018; 1: 127- 36. https://dx.doi.org/10.17060/ijodaep.2018.n1.v3.1233

15. Vázquez C, Duque A, Hervás G. Satisfaction with life scale in a representative sample of Spanish adults: Validation and normative data. Span J Psychol 2013; 16: E82. https:// dx.doi.org/10.1017/sjp.2013.82 
16. Musiol K, Bulska W, Brożek P, Oślizło B, Ryzak S, Dubiel J, Sobol-Milejska G. Quality of life in survivors of childhood brain tumour and the association of children's diseases on quality of their parents life. Psychooncology 2019; 28: 1088-95. https://dx.doi.org/ 10.1002/pon.5061

17. Faith MA, El-Behadli AF, Frazee LA, Pratt CD, Stromberg S. Parents' emotion socialization beliefs moderate relations between parent and patient coping, but not sibling coping, with pediatric cancer. Psychooncology 2019; 28: 1559-66. https://dx.doi. org/10.1002/pon.5132

18. Espada MC, Grau C. Estrategias de afrontamiento en padres de niños con cáncer. Psicooncologia 2012; 9: 25-40. https://dx.doi.org/10.5209/rev_PSIC.2012.v9.n1.39136

19. Matos D, Mercerón Y. Potenciación de la capacidad de resiliencia en familiares de niños con cáncer mediante un programa de intervención psicosocial. Medisan 2017; 21: 36170.

20. Navea A, Tamayo JA. Características de la resiliencia familiar en pacientes oncológicos pediátricos: una revisión sistemática. Psicooncología 2018; 15: 203-16. https://dx.doi. org/10.5209/psic.61431

21. Luo D, Eicher M, White K. Resilience in adult cancer care: A review and concept analysis. Ann Oncol 2018; 29: 132-8. https://dx.doi.org/10.1093/annonc/mdy444.012

22. Pintado S, Cruz MF. Factores de resiliencia en niños y adolescentes con cáncer y su relación con el género y la edad. Ciencia Ergo Sum 2017; 24: 137-44. https://dx.doi. org/10.30878/ces.v24n2a5

23. Heilemann MV, Lee K, Kury FS. Psychometric properties of the Spanish version of the Resilience Scale. J Nurs Meas 2007; 11: 61-72. https://dx.doi. org/10.1891/106137403780954976

24. Streisand R, Braniecki S, Tercyak KP, Kazak AE. Childhood illness-related parenting stress: the pediatric inventory for parents. J Pediatr Psychol 2001; 26: 155-62.

25. Del Rincón C, Remor E, Arranz P. Estudio psicométrico preliminar de la versión española del Pediatric Inventory for Parents (PIP). Int J Clin Health Psychol 2007; 7: 435-52. Disponible en: http://www.redalyc.org/articulo.oa?id=33717060012

26. Pavot W, Diener E. Review of the Satisfaction With Life Scale. Psychol Assess 1993 ; 5 : 164-72.

27. Harper FW, Peterson AM, Albrecht TL, Taub JW, Phipps S, Penner LA. Satisfaction with support versus size of network: Differential effects of social support on psychological distress in parents of pediatric cancer patients. Psychooncology 2016; 25: 551-8. https:// dx.doi.org/10.1002/pon.3863

28. Liu Y, Wang Z, Lü W. Resilience and affect balance as mediators between trait emotional intelligence and life satisfaction. Pers Individ Differ 2013; 54: 850-55. https://dx.doi. org/10.1016/j.paid.2012.12.010

29. Calhoun LG, Tedeschi RG. The foundations of posttraumatic growth: An expanded framework. En: Calhoum LG, Tedeschi RG, editors. Handbook of posttraumatic growth. Mahwah, New York: Lawrence Erlbaum Associate Publishers, 2014; p. 3-23.

30. Guil R, Zayas A, Gil-Olarte P, Guerrero C, González S, Mestre JM. Bienestar psicológico, optimismo y resiliencia en mujeres con cáncer de mama. Psicooncología 2016; 13: 127. https://dx.doi.org/10.5209/rev_PSIC.2016.v13.n1.52492

31. Ochoa C, Casellas-Grau A, Vives J, Font A, Borràs, JM. Positive psychotherapy for distressed cancer survivors: Posttraumatic growth facilitation reduces posttraumatic stress. Int J Clin Health Psychol 2017; 17: 28-37. https://dx.doi.org/10.1016/j.ijchp.2016.09.002 
32. Martín A, Tamayo JA. Promoción de la resiliencia familiar en oncología pediátrica: Una revisión sistemática. Revista Clínica Contemporánea 2018; 9, e22, 1-13. https://dx.doi. org/10.5093/cc2018a18

33. Sánchez-Egea R, Rubio-Aparicio M, Sánchez-Meca J, Rosa-Alcázar AI. Psychological treatment for family members of children with cancer: A systematic review and metaanalysis. Psychooncology 2019; 28: 960-9. https://dx.doi.org/10.1002/pon.5052

34. Kedia SK, Collins A, Dillon PJ, Akkus C, Ward KD, Jackson BM. Psychosocial interventions for informal caregivers of lung cancer patients: A systematic review. Psychooncology 2020; 29: 251-62. https://dx.doi.org/10.1002/pon.5271

35. Monteagudo Y, Vicente A, Peralta MI. Estudio de la resiliencia en pacientes oncológicos de la AECC en Castellón. Àgora Salut 2016; 3: 257-265. https://dx.doi.org/10.6035/ agorasalut.2016.3.27

36. González-Arratia N, Valdez JL. Resiliencia: diferencias por edad en hombres y mujeres mexicanos. Acta Invest Psicol 2013; 3: 941-55.

37. Fínez MJ, Morán MC. La resiliencia y su relación con salud y ansiedad en estudiantes españoles. Int J Dev Educ Psychol 2015; 1: 409-16. https://doi.org/10.17060/ijodaep.2015. n1.v1.90

38. Ramírez-Granizo I, Castro-Sánchez M. Análisis de los niveles de resiliencia en función del género y factores del ámbito educativo en escolares. ESHPA - Education, Sport, Health and Physical Activity 2018; 2: 50-61. [Acceso 1 de abril de 2021]. Disponible en: https://hdl.handle.net/10481/48262

39. Prado R, Águila M. Diferencia en la resiliencia según género y nivel socioeconómico en adolescentes. Persona 2003; 6: 179-196. [Acceso 1 de abril de 2021]. Disponible en: httpss://www.redalyc.org/articulo.oa?id=147118110009

40. García-Calvente MM, Mateo-Rodríguez I, Maroto-Navarro G. El impacto de cuidar en la salud y la calidad de vida de las mujeres. Gac Sanit 2004; 18: 83-92. https://dx.doi. org/10.1157/13061998 